5. N. Jacobson, Structure of rings, Amer. Math. Soc. Colloquium Publications, vol. 37, 1956.

6. F. Kasch, Über den Endomorphismring eines Vektoraumes und den Satz von der Normal basis, Math. Ann. vol. 126 (1953) pp. 447-463.

7. T. Nakayama, Normal basis of a quasi-field, Proc. Imp. Acad. Tokyo vol. 16 (1940) pp. 532-536.

8. - Galois theory of simple rings, Trans. Amer. Math. Soc. vol. 73 (1952) pp. 276-292.

9. S. Perlis, Normal bases of cyclic fields of prime power degree, Duke Math. J. vol. 9 (1942) pp. 507-517.

The Pennsylvania State University

\title{
REMARK ON AUTOMORPHISMS OF GROUPS
}

\section{MAURICE AUSLANDER}

Let $G$ be a group with center $C$. Let $\alpha$ be an automorphism of $G$ and $n$ an integer such that $\alpha^{n}$ is an inner automorphism. Thus there is a $g$ in $G$ such that $\alpha^{n}(x)=g x g^{-1}$ for all $x$ in $G$. Applying $\alpha$ to both sides of this equation we have that $\alpha^{n}(\alpha(x))=\alpha(g) \alpha(x) \alpha(g)^{-1}$ for all $x$ in $G$. Since every element in $G$ can be written as $\alpha(x)$ for some $x$ in $G$, it follows that $g$ and $\alpha(g)$ induce the same inner automorphism of $G$. Thus $g^{-1} \alpha(g)=c$ where $c$ is in $C$. Now if $y$ is in $C$, then $(g y)^{-1} \alpha(g y)$ $=g^{-1} y^{-1} g c \alpha(y)=c y^{-1} \alpha(y)$. Thus as $x$ runs through all $x$ in $G$ which induce the inner automorphism $\alpha^{n}$, the elements of the form $x^{-1} \alpha(x)$ run through the entire $\operatorname{coset} c C_{\alpha}$ in $C / C_{\alpha}$, where $C_{\alpha}$ is the subgroup of $C$ consisting of all elements of the form $y^{-1} \alpha(y)(y$ in $C)$. This element of $C / C_{\alpha}$ depends on $n$ and will be denoted by $o(\alpha, n)$.

Theorem. If all the fixed points of $\alpha$ are in the center of $G$, then $\alpha^{n^{2}}=1$. Further $\alpha^{n}=1$ if and only if $o(\alpha, n)=(1)$.

Proof. Let $g$ in $G$ induce the inner automorphism $\alpha^{n}$. Then by the previous remarks we have that $g^{-1} \alpha(g)=c$ where $c$ is in $C$. Thus the abelian subgroup of $G$ generated by $C$ and $g$ is stable under $\alpha$. Since $\alpha^{n}(g)=g$, it follows that $\prod_{i=0}^{n-1} \alpha^{i}(g)$ is a fixed point of $\alpha$ and is thus in $C$. On the other hand, since $\alpha(g)=g c$, we have that $\prod_{i=0}^{n-1} \alpha^{i}(g)$ $=g^{n} d$ for some $d$ in $C$. Therefore $g^{n}$ is in $C$ which means that $\alpha^{n^{2}}=1$.

It is clear that if $\alpha^{n}=1$, then $o(\alpha, n)=(1)$. Suppose $o(\alpha, n)=(1)$. Then by our introductory remarks, we can choose a $g$ in $G$ such that $g$ induces the inner automorphism $\alpha^{n}$ and $g^{-1} \alpha(g)=1$. Thus $g$ is a fixed point of $\alpha$. Consequently $g$ is in the center of $G$, which means that $\alpha^{n}=1$.

Received by the editors September 17, 1957. 
It should be observed that if $o(\alpha, n)=c C_{\alpha}$, then $c$ has the property that $\prod_{i=0}^{n-1} \alpha^{i}(c)=1$. Thus $o(\alpha, n)$ is actually an element of the cohomology group $H^{3}\left(Z_{n}, C\right)$, where $Z_{n}$ is the integers $\bmod n$ and a generator of $Z_{n}$ operates on $C$ as $\alpha$ does. It is easily seen that $o(\alpha, n)$ is the "obstruction" in the sense of Eilenberg and MacLane of the $Q$-kernel $Z_{n} \rightarrow A(G) / I(G)$ given by $m+Z \rightarrow \alpha^{m} I(G)$, where $A(G)$ and $I(G)$ are the automorphism and inner automorphism groups of $G$ respectively [1]. Thus the above theorem gives another interpretation of the "obstruction" in this special case.

\section{REFERENCE}

1. S. Eilenberg and S. MacLane, Cohomology theory in abstract groups, II. Ann. of Math. vol. 48 (1947) pp. 326-341.

BrandeIS UNIVERSITY 